\title{
Another mountain from a molehill
}

Stealing ideas is bad for research and the reputation of science, but so are unfounded allegations that there has been a theft when there is none, now put in circulation by a materials scientist from Pennsylvania.

Plagiarism is the most serious of the known crimes against scholarship. Not only does it confuse the record by making it impossible to tell who exactly said what, but it amounts to the literal theft of another's words, thereby depriving the victim not merely of the credit for the content of the stolen words but of whatever thought and imagination they embody. It is a shabby business.

So what about the appropriation of others' ideas? That is also shabby, whence the convention that authors should be meticulous in their references to the antecedents of their research. By failing to refer to a significant earlier article, an author misleads his readers about the genesis of what he or she has to say and also captures an undue proportion of the credit for what is new. Oversights of this kind, deliberate or otherwise, are the most common cause of disputes about priority. People will even hotly claim precedence for ideas recognized to be hare-brained, but even that is understandable.

In short, the omission of crucial references is reprehensible, may amount to the theft of other people's ideas and, as far as possible, should be stopped. The excuse that 'there is no copyright in ideas' will not wash. But there are obvious difficulties. What about, for example, when two groups of people make more or less the same discovery at more or less the same time and submit accounts for publication separately? One may accuse the other of having picked up crucial information at a meeting, or may even suspect that referees have spilled the beans.

Obviously, some oversights can also be innocently explained; an author may not have known of earlier related work. A recent article on this page (Nature 349,$363 ; 1991$ ) which drew attention to a recent reassessment of the importance of the expansion of the Universe and its age in the resolution of Olbers' paradox has, for example, already drawn the comment that a similar conclusion was reached in a contribution to Nature nearly 30 years ago.

Such incidents, while still regrettable, are likely to become more common. However the bibliographic databanks are embellished, there is a high risk that anticipatory work will be overlooked, at least in fields not constantly reworked by the textbook and monograph writers. As the record on which contemporary research is built stretches further back, such occurrences will multiply, at least until the bibliographers learn to index their material by concept as well as content.

Most people accept these difficulties but not, it appears, Professor D. M. ('Rus- tum') Roy of the Materials Research Laboratory at the Pennsylvania State University, who is well-known as a proselytizer of materials science. Roy is now deeply offended that Nature has published an article that fails to refer to two earlier publications (also in Nature) in 1971 and 1974 with which he had been associated. Ordinarily, the first step in such a saga is a letter of protest to the offending journal, which, fair play, Roy sent on 13 March, but intemperately. Most of what follows is concerned with what has happened since.

The article about which Roy protests is that by Patricia A. Bianconi, Jun Lin and Angela R. Strzelecki (Nature 315, 349; 24 January 1991). Piquantly, it may be thought, the authors are also at Pennsylvania State University, but in the chemistry department. They point out the potential importance of synthetic materials in which inorganic constitutents are precipitated within an organic matrix and describe the precipitation of CdS crystals within a synthetic organic matrix consisting of polyethylene-oxide.

Nobody would mistake the article for a claim to have made a usable material; its chief interest, as explained by Stephen Mann in an accompanying article (Nature 349, $285 ; 1991)$ is that the authors have been able to find conditions under which apparently amorphous $\mathrm{CdS}$ makes a phase transition to a cubic form, suggestive of how the mineral components in materials such as bone and teeth are often orientated and otherwise disposed so as to enhance their links with the underlying matrix. Appropriately, but, as events have shown, perhaps unfortunately, the authors use the terms 'biomimetic' and 'biocomposite'.

Roy has taken up the cudgels on behalf of two earlier articles. One describes the formation of porous bone-like structures by using a resin to make a cast of the pores in natural bony material (the skeleton of the echinoderm known as the slate-pencil urchin) in which ceramic materials are then precipitated (J. N. Weber et al. Nature 233, 337; 1971). The other describes a technique for replacing the carbonate of coral by phosphate to form harder apatite (D. M. Roy \& S. K. Linnehan Nature 247, 220; 1974). Both principles have since been used in the fabrication of synthetic composites.

Roy's cudgels have been exercised energetically. He has, for example, distributed widely an undated memorandum "in the interests of maintaining the integrity of the modern science enterprise" which says that, while this is not a case of "cheating, fraud, malice, etc.", Nature and the Pennsylvania State University have acted jointly in the overselling of science, which is being advertized "like cigarettes using inaccurate misleading exaggerations". The same document complains that Nature made things worse by inviting Stephen Mann to comment on Bianconi et al.

This would ordinarily have been but a storm in a teacup had not the document fallen into the hands of a news magazine to which Roy confided that he knew from a telephone conversation that the editor of Nature, correctly identified by first name, was annoyed ("pissed off" was the verb he chose) with Mann for having written an egregious puff, but that Roy did not know whether the outcome would be a scandal or a "cover-up". Yet in the only conversation on this or (if memory serves) any other subject, Roy said merely "Why would I say a thing like that?" It is mystifying, to say the least.

So is the feasibility of the remedies Roy advocates. He would, for example, require all publications to include an explicit statement of how the authors had searched the literature, that journals should be compelled to publish amendments to lists of references and that grant-making agencies should appoint ombudsmen to assess the validity of claims to novelty. (One little irony is that the two earlier articles do not use the word 'biomimetic', on which a literature search would have been conducted, but which may not have been invented in the 1970s.) There was even at one stage talk of having this apparatus given teeth (in the United States) by an act of Congress.

All of us will appreciate, all too often from first-hand experience, the acuteness of the sense of neglect evoked by recognizing our own thoughts converted by others into words, and the ferocity of the emotions that can be aroused when others occupy intellectual territory we regard as ours. (This, no doubt, explains Roy's assertion that using the word 'biomaterials' in connection with the precipilation of $\mathrm{CdS}$ in a polymer gel is tantamount to "poisoning the language of science'.) Yet even by those tests, Roy's reaction to Bianconi is no less exaggerated than the supposed overselling of which he complains.

Consciously appiopriating the ideas of others without acknowledgement is bad for science, but so is making a needless fuss about empty issues. Indeed, gratuitous unseemliness may be just as damaging, suggesting to others that researchers are incorrigibly pelty and quarrelsome. John Maddox 